\title{
A Multi-bit/cell PUF Using Analog Breakdown Positions in CMOS
}

\author{
K.-H. Chuang ${ }^{1,2}$, E. Bury ${ }^{2}$, R. Degraeve², B. Kaczer², T. Kallstenius ${ }^{1,2}$, G. Groeseneken²,3, \\ D. Linten ${ }^{2}$ and I. Verbauwhede ${ }^{1}$ \\ ${ }^{1}$ imec-COSIC, KU Leuven, Leuven, Belgium \\ ${ }^{2}$ imec, Leuven, Belgium \\ ${ }^{3}$ ESAT, KU Leuven, Belgium \\ Email: Kai.Hsin.Chuang@imec.be
}

\begin{abstract}
A physically unclonable function (PUF) utilizing the analog positioning of breakdown spots in CMOS transistors is presented. In contrast to digital positioning based on a threetransistor cell [3], this new approach has the capability of generating multiple bits from a more compact two-transistor cell. The basic properties and reliability aspects of this PUF are studied based on the test chips fabricated in a commercial $40 \mathrm{~nm}$ CMOS technology. The breakdown positions in high density arrays have been characterized, proving that indeed multiple bits can be generated from a single nFET. Through consecutive measurements, the long-term stability is found to be reduced, due to shrinking of readout window, especially when more bits are generated. Finally, high temperature also negatively impact the stability, indicating that the analog BD-PUF is a lesser promising candidate for PUF application than the previously presented binarized design.
\end{abstract}

Index Terms - hardware security, cryptography, physically unclonable function, reliability, oxide breakdown

\section{INTRODUCTION}

A Physically Unclonable Function (PUF) in the silicon technologies is an essential security primitive for on-chip key generation and entity authentication applications [1]. A silicon PUF utilizes the inherent and uncontrollable process-related variations on the devices or interconnections, to extract a random data pattern. Ideally this pattern is unique for all the chips that are identically fabricated and is reproducible in various noisy conditions. The conventional PUF implementations such as SRAM PUFs [2], however, may have less unique data patterns due to systematic mismatches, and the unprocessed PUF data are less reproducible due to its high intrinsic sensitivity to noise.

In order to improve the quality of PUF, one of the recent PUF implementations uses the binarized breakdown position in two nFETs as the source of entropy, so called binary BDPUF [3]. It has been proven in silicon that this proposed implementation satisfies all the PUF requirements including randomness, uniqueness and stability. Moreover, this earlierproposed implementation has also been shown to survive the radiation environment of the low-orbit space applications [4].

Following the same concept of utilizing oxide breakdown position randomness as entropy source, we propose a new
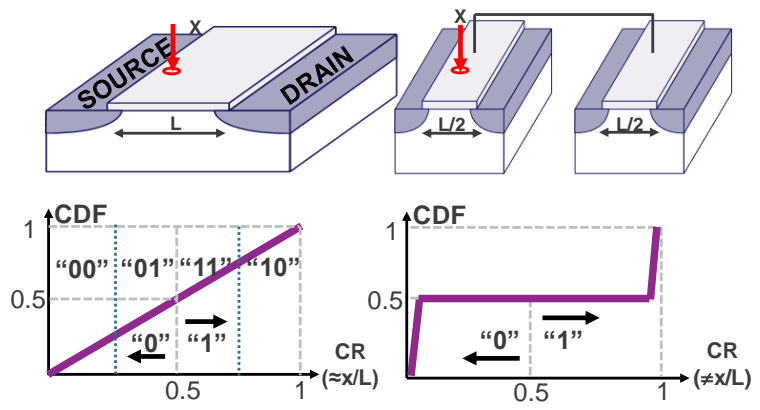

Fig. 1 Schematic comparison of the difference between the single nFET and double nFET BD-PUF cell. The resulting current ratio (CR) is a uniform-like distribution and a binary-like distribution [3] respectively.

The uniform distribution can produce more than one bit per unit cell.

compact structure that generates multiple bits per unit cell, by extracting its exact (analog) breakdown position, namely, analog BD-PUF. As illustrated in Fig. 1, this technique can provide multi-bit entropy in a unit cell whereas the binary BDPUF is limited to only one-bit per unit cell. Since the entropy in a key stream determines the level of security, the potential of generating multiple bits has the advantage of providing a higher level of security with a limited amount of PUF cells.

This paper is organized as follows: in Section II, we discuss the concept of analog breakdown positions and the dedicated test structure. In Section III, we show the experimental results from the fabricated PUF arrays. Section IV discusses the properties of the PUF.

\section{CONCEPT AND TEST STRUCTURE OVERVIEW}

The analog BD-PUF cell contains one minimum-width nFET transistor, utilized for the breakdown, and a pFET selector, as shown in Fig. 2(a). Similar to [3], this PUF cell requires a forming step to generate a soft-breakdown path in the gate oxide of the nFET. Forming is done by stressing the nFET gate with a high voltage through the pFET selector. As soon as the nFET breaks, the pFET selector acts as a current compliance that limits the breakdown hardness. Moreover, once breakdown in the nFET is present, the selector also induces a voltage drop which reduces the stress of the nFET 
(a)

(c)

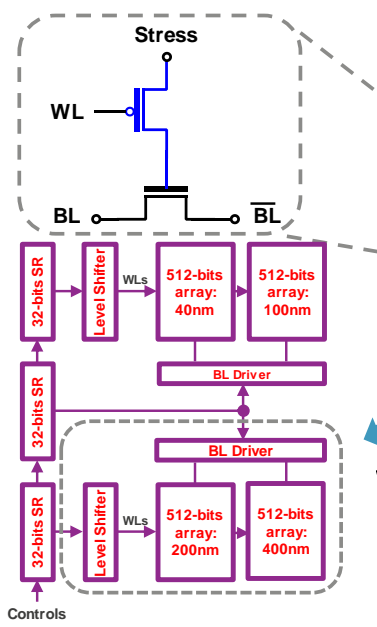

(b)

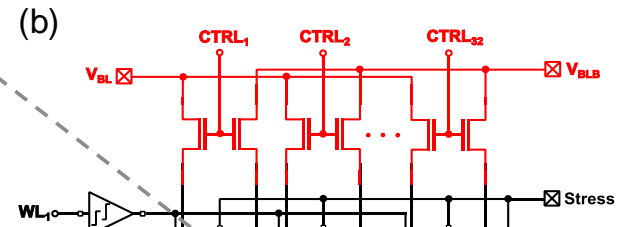

(d)
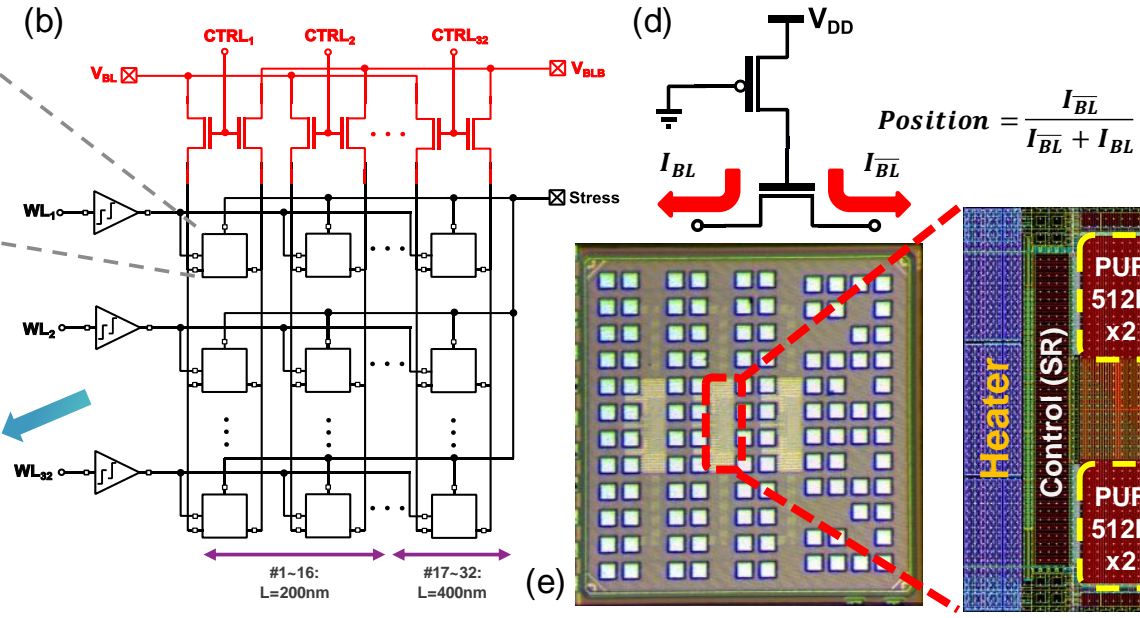

Fig. 2 (a) Unit cell of the single nFET BD-PUF, consisting of one minimum width nFET (120nm x L) and a pFET selector. (b) The test array with $2 \mathrm{x}$ 512 cells, which has been fabricated in a commercial CMOS process. (c) The complete circuit diagram with all periphery circuits, the dashed box is equivalent to the array in (b). (d) BD-PUF cell under measurement (read-out) condition, BD position is extracted by s-ratio formula [7]-[9]. (e) Chip mircrograph and the core layout. All the PUF arrays are surrounded by the poly-heaters which enables the localized high-temperature measurement.

gate oxide, preventing multiple breakdown spots to occur in the same oxide. Since the test structure is fabricated in a process identical to the binary BD-PUF, the TDDB behavior during the forming step is identical to [3] and therefore not further discussed in this paper.

\section{A. Test structure in 40nm CMOS}

The test structure is designed and fabricated in a commercial 40nm CMOS process, containing four 512-bit PUF arrays with gate lengths of 40, 100, 200 and 400nm respectively, as shown in Fig. 2 (c). The layout and the chip micrograph are shown in Fig. 2(e), where the PUF arrays are surrounded by metallic heating stripes that enable fast and localized high-temperature generation and measurements [5]. In this test structure with increased amount of cells comparing to [3], all the word and bit-lines remain accessible and are controlled by the peripheral circuits. By providing a sequence of control signals to the periphery, we are able to measure analog current characteristics of every single cell, before, during or after the forming step. Consequently, this test structure can be utilized to provide statistical reliability characterization such as in [6], in particular for TDDB.

\section{B. Readout technique}

Similar to the binarized BD-PUF, the analog breakdown characteristics of every PUF cells can be read out using the $s$ ratio technique [7]-[9], which is schematically illustrated in Fig. 2 (d). Applying this s-ratio equation, the results are more likely to be uniformly distributed as shown in Fig. 3, which represents the lateral breakdown position within nFETs. Depending on the number of quantization levels, the analog breakdown positions can be extracted to different numbers of bits. The extracted bits will have no bias [1] if the breakdown spots are uniformly distributed. This is valid when the transistors are stressed under inversion mode [10].

\section{EXPERIMENTAL RESULTS}

In order to measure the breakdown positions, the PUF arrays are all formed with a $4.2 \mathrm{~V}$ stress for 10 seconds, and the compliance current is set as $100 \mu \mathrm{A}$, which is controlled by the pFET selector. The nFETs are operated in strong inversion for both the forming and the measurement phase.

\section{A. Breakdown positions}

The measured current ratio distributions, indicating the position of the soft-breakdown path along the channel, are
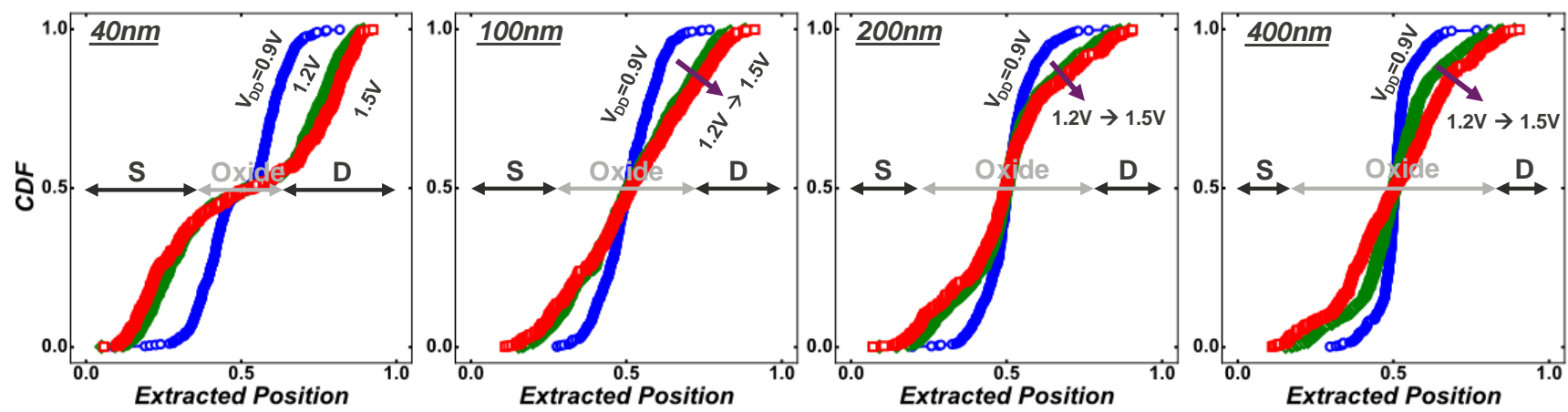

Fig. $3 \mathrm{CDFs}$ of the extracted position under three $V_{D D}$. The inset shows the resulting distribution shape is affected by the gate length. The PUF stability of each gate-length can be predicted using the distribution shape and the BD model described in [11]. 


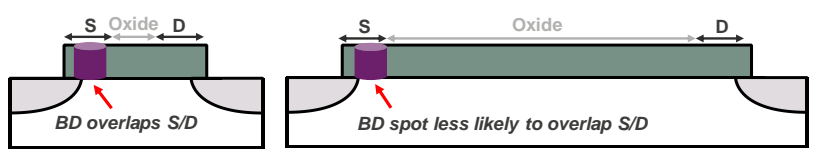

Fig. 4 The effect of the source/drain overlaps of breakdown spots. With shorter gate length, the breakdown spots are more likely to overlap with $\mathrm{S} / \mathrm{D}$, resulting in a more binarized distribution.

measured at multiple $V_{D D}$ conditions and shown in Fig. 3. The distributions are not the ideal uniform distributions, but the probability of having a current ratio below or above 0.5 is close to $50 \%$ in all cases and regardless of the read-out voltage, which is indeed a signature of good randomness.

Based on the bending of the current ratio distributions, one can discriminate three different regions. The existence of these regions can be attributed to the overlapping with the source or drain areas. As illustrated in Fig. 3 and Fig. 4, the points in different regions correspond to the breakdown spots which are overlapping with the source(S)/drain(D) area, or having no overlap with both side (oxide). The spots overlapping with $\mathrm{S} / \mathrm{D}$ have stronger current difference since the current does not have to pass the channel. Hence the resulting current ratios are not representative for their actual positions. This is further confirmed by studying the breakdown locations in the nFETs with shorter gate length are more likely to have S/D overlap, and hence the current ratio distributions become more 'binarized'.

Comparing to the binary BD-PUF [3], the voltage dependence of the analog BD-PUF is less obvious especially for $\mathrm{V}_{\mathrm{DD}}$ above $1.2 \mathrm{~V}$, since the voltage dependence on tunneling current will equally affect both source and drain current. However, the distributions become narrower at lower voltage $\left(\mathrm{V}_{\mathrm{DD}}=0.9 \mathrm{~V}\right)$, which can be explain by (1) with the presence of offset current from other devices sharing the same bit-line. The resulting current ratio becomes closer to 0.5 as the current flow through the oxide decreases, which gives a negative impact on BD-position extraction.

$$
\text { Ratio }=\frac{I_{\overline{B L}}+I_{o f f s e t}}{I_{B L}+I_{o f f s e t}+I_{\overline{B L}}+I_{o f f s e t}}
$$

\section{B. Binarized breakdown positions and the stability:}

In order to generate the key stream for cryptographic applications, the PUF responses are usually digitized into binary numbers for the post processing. The simplest case is to binarize the extracted breakdown positions to " 0 " and " 1 " with a decision threshold of 0.5. As shown in Fig. 5 (a), there is no pattern observed and the ratio of " 0 " and " 1 " bits are almost equal, indicating a good randomness of the binarized breakdown position. Note that if only one quantization level is defined, the analog BD-PUF behaves as a conventional PUF with single-bit/cell. This implementation is, however, less reliable compared to the binary BD-PUF, since it is more difficult to distinguish " 0 " and " 1 ".

Since no dedicated readout circuit is available, the stability of the PUF readout is estimated by repeating the DC-current measurement multiple times with $\mathrm{V}_{\mathrm{DD}}$ equals to $1.5 \mathrm{~V}$. Even though the actual BD-position will not change over time, the

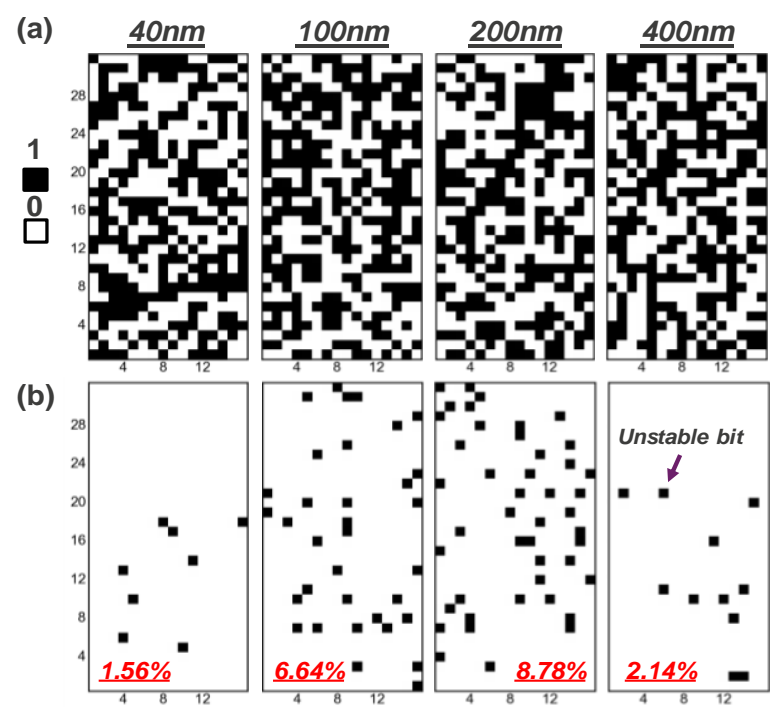

Fig. 5 (a) The binarized data for the four 512-bit array with different gate length, showing no pattern. (b) The unstable PUF cells that have bit-flips during 5 measurements. The red-colored numbers in the figure indicate the percentage of the unstable cells in the array.

position, readout and extracted using current ratio, can be affected by measurement noise, especially at lower $V_{D D}$. For some devices that have a BD position close to the center of the device, the resulting current ratio might switch across the threshold, resulting in bit-flipping after digitization. Fig. 5(b) shows the unstable bits and the percentage in four PUF arrays with different gate length. The unstable bits are defined as the bits that flip at least once during five consecutive measurements. Although these numbers may still increase with more measurement cycles, the results from five measurements are sufficient for qualitative comparisons.

In the case of generating a single bit, the array with the minimum gate length has the best stability among all. This is the consequence of the higher probability to have breakdown spots overlapping with S/D as observed in Fig. 3, i.e. more binarized. The stability is degraded as the gate length increases to $100 \mathrm{~nm}$, because the breakdown spot is less likely to overlap the terminals and the current ratio becomes more uniform. This trend, however, does not continue since the breakdown spot is less likely to be located right at the middle as the gate length is further increased. Consequently, the PUF array of 400nm gate length has the second-best stability.

\section{FEASIBILITY STUDY}

The feasibility of the proposed analog BD-PUF is studied mainly on its capability to provide more than one PUF bits per cell. The thermal stability, a general requirement for PUFs, is also examined in this section.

\section{A. Multiple bits generation}

As illustrated in Fig. 1, the breakdown position of the analog BD-PUF can be digitized into more bits to harvest more entropy. Taking the PUF with the gate length of $40 \mathrm{~nm}$ as an example, the breakdown position is divided into four regions, by the decision thresholds of $0.25,0.5$ and 0.75 . These positions are encoded into the 2-bit Gray codes as in 

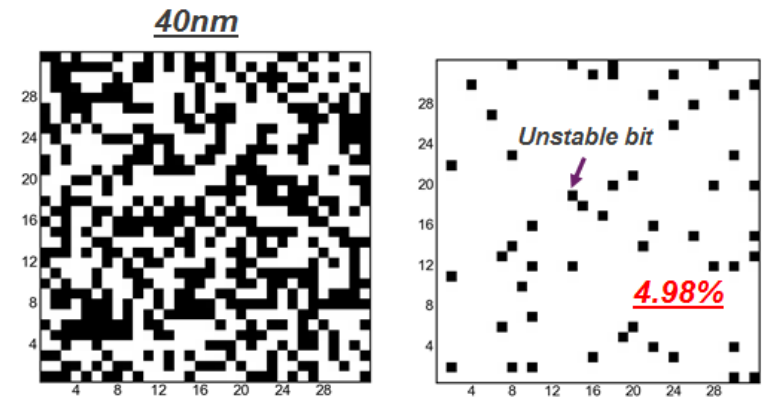

Fig. 6 The 2b/cell encoded data from the 512-bit PUF array with gate length of $40 \mathrm{~nm}$. The number of the unstable bits is significantly

Fig. 1. The resulting data with the doubled number of bits are shown in Fig. 6. Since the deltas of each quarter are not equal, the resulting randomness is degraded. The normalized Hamming distance between the original 512-bit response and the additional 512-bit response is equal to 0.527 (ideal: 0.5 ). This indicates a weak correlation with the original response, and hence the entropy is sufficiently increased but not perfectly doubled by generating two bits per unit cell.

Moreover, as the number of decision thresholds is increased, more devices may have a breakdown spot close to the thresholds. Hence the percentage of unstable cells will increase with the number of bits per cell. A clear stability degradation can be observed in Fig. 6. The percentage of the unstable bits in the 512-bit PUF array with 40nm gate length is about three times higher, which corresponds to the three decision thresholds.

Comparing to the binary BD-PUF, the proposed analog BD-PUF seems to have the ability to generate the same amount of PUF bits with less area. However, this advantage might not be valid due to the bit instability of PUF cells. In practical applications, in order to correct the bit-errors, more PUF cells along with complicate error correction logics are required, which can be in total several times larger than the binary BD-PUF with perfect bit stability.

\section{B. Thermal stability}

As an important reliability aspect, the thermal stability of the proposed analog BD-PUF is also studied. Similar to [3], the on-chip poly-heaters are implemented to check the instantaneous local heating of the PUFs. In order to heat up the entire PUF array, the poly-heaters are enlarged to surround the PUF arrays, as shown in Fig. 2(e). The poly-heaters have a power to temperature relation calibrated by a temperature sensing diode and external heating [3][5], as shown in Fig. 7.

The extracted positions at room temperature and $400 \mathrm{~K}$, heated-up by the heaters, are plotted in Fig. 8, showing that the extracted BD-position is highly sensitive to temperature. This result differs from the results shown in Fig. 7 (b) [3], where the increase of temperature has no impact. This can be understood as follows: The high temperature sensitivity of the channel conductance impacts the BD position determination (through the s-ratio formula) in the case of analog BD-PUF, while in the binary BD-PUF [3] only the temperatureinsensitive tunnel current through the oxide plays a role [12]. The bit-flipping caused by temperature variation has an even
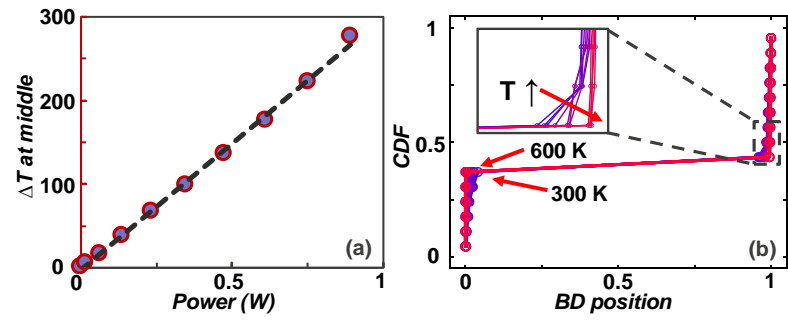

Fig. 7(a)The temperature in the middle raised by Joule Heating v.s. power. (b) The temperature dependence of the binary BD-PUF in [2], showing a good thermal stability.

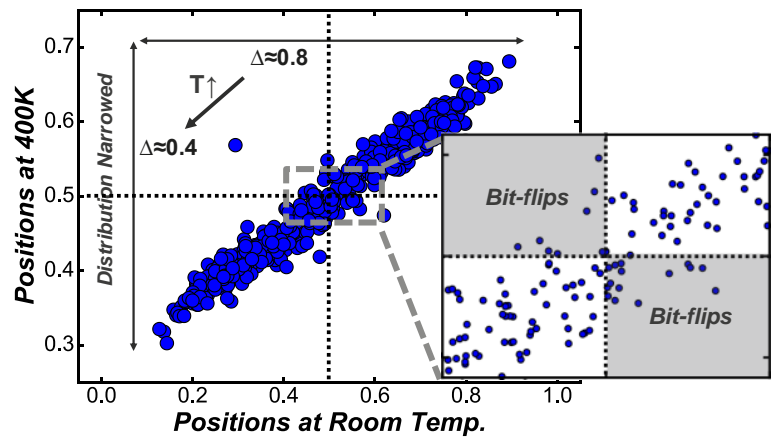

Fig. 8 The relation between extracted position under room temperature and $400 \mathrm{~K}$ (length $=40 \mathrm{~nm})$. The inset shows there are several bit-flips. This is not the same array as in Fig.3.

stronger negative impact, since it originates internally and hence cannot be improved by readout circuitry.

\section{CONCLUSION}

The feasibility of extracting the entropy from random breakdown position within a single nFET was tested in 40nm CMOS technology. This usually detrimental reliability phenomenon was proved to provide multiple PUF bits per unit cell. Apart from this advantage, it also necessitates a more precise readout scheme and suffers from poor bit stability especially at elevated temperature. Consequently, the analog BD-PUF will need a significant improvement on the bitstabilization techniques to be able to replace the binary BDPUF.

\section{REFERENCES}

[1] R. Maes, "Physically unclonable functions: Construction, properties and applications", PhD Thesis, KU Leuven, 2012.

[2] D. E. Holcomb, W. P. Burleson and K. Fu, "Power-Up SRAM State as an Identifying Fingerprint and Source of True Random Numbers," in IEEE Transactions on Computers, vol. 58, no. 9, pp. 1198-1210, Sept. 2009.

[3] K. H. Chuang et al., "Physically unclonable function using CMOS breakdown position," 2017 IEEE International Reliability Physics Symposium (IRPS), Monterey, CA, 2017, pp. 4C-1.1-4C-1.7.

[4] P. F. Wang et al., "X-ray and Proton Radiation Effects on $40 \mathrm{~nm}$ CMOS Physically Unclonable Function Devices," in IEEE Transactions on Nuclear Science, in press.

[5] J. Franco, B. Kaczer and G. Groeseneken, "Poly -Si heaters for ultra fast local temperature control of on -wafer test structures", Microelectronic Engineering, Vol. 114, pp. 47 -51, (2014)

[6] E. Bury et al., "Statistical assessment of the full $V_{G} / V_{D}$ degradation space using dedicated device arrays," 2017 IEEE International 
Reliability Physics Symposium (IRPS), Monterey, CA, 2017, pp. 2D5.1-2D-5.6.

[7] R. Degraeve, B. Kaczer, A. De Keersgieter and G. Groeseneken, "Relation between breakdown mode and location in short-channel nMOSFETs and its impact on reliability specifications," in IEEE Transactions on Device and Materials Reliability, vol. 1, no. 3, pp. 163-169, Sep 2001

[8] M. A. Alam, D. Varghese and B. Kaczer, "Theory of Breakdown Position Determination by Voltage- and Current-Ratio Methods," in IEEE Transactions on Electron Devices, vol. 55, no. 11, pp. 31503158, Nov. 2008.

[9] E. Bury et al., "Study of (correlated) trap sites in SILC, BTI and RTN in SiON and HKMG devices," Proceedings of the 21th International Symposium on the Physical and Failure Analysis of Integrated Circuits (IPFA), Marina Bay Sands, 2014, pp. 250-253.
[10] B. Kaczer, R. Degraeve, A. De Keersgieter, K. Van de Mieroop, V. Simons and G. Groeseneken, "Consistent model for short-channel nMOSFET after hard gate oxide breakdown," in IEEE Transactions on Electron Devices, vol. 49, no. 3, pp. 507-513, Mar 2002.

[11] B. Kaczer, R. Degraeve, F. Crupi, A. De Keersgieter and G. Groeseneken, "Understanding nMOSFET Characteristics after Soft Breakdown and Their Dependence on the Breakdown Location," 32nd European Solid-State Device Research Conference, 2002, pp. 139-142.

[12] R. Degraeve, B. Govoreanu, B. Kaczer, J. Van Houdt and G. Groeseneken, "Measurement and statistical analysis of single trap current-voltage characteristics in ultrathin SiON," IEEE International Reliability Physics Symposium, 2005. Proceedings. 43rd Annual., pp. 360-365. 